\title{
Piezoelectric energy harvesting from traffic-induced bridge vibrations
}

\author{
Michaël Peigney ${ }^{1}$, Dominique Siegert ${ }^{2}$ \\ ${ }^{1}$ Université Paris-Est, Laboratoire Navier (Ecole des Ponts ParisTech, IFSTTAR, \\ CNRS), F-77455 Marne-la-Valle Cedex 2, France \\ ${ }^{2}$ Université Paris-Est, IFSTTAR/COSYS/LISIS, F-77455 Marne-la-Valle Cedex 2, \\ France \\ E-mail: michael.peigney@polytechnique.org
}

\begin{abstract}
This paper focuses on energy harvesting of traffic-induced vibrations in bridges. Using a pre-stressed concrete highway bridge as a case study, in situ vibration measurements are presented and analyzed. From those results, a prototype of cantilever piezoelectric harvester was designed, tested, and modeled. Even though the considered bridge vibrations are characterized by small amplitude and a low frequency (i.e. below $15 \mathrm{~Hz}$ ), it is shown that mean power of the order of $0.03 \mathrm{~mW}$ can be produced, with a controlled voltage between 1.8 and $3.6 \mathrm{~V}$. A simple model is proposed for the theoretical prediction of the delivered power in terms of traffic intensity. That model shows good agreement with the experimental results and leads to a simple but effective design rule for piezoelectric harvesters to be used on bridges.
\end{abstract}




\section{Introduction}

This paper focuses on using bridge vibrations as an energy source for self powered health monitoring sensor nodes. As traffic-induced bridge vibrations are low frequency and have small amplitudes, energy harnessing is challenging even for measuring slow time varying signal such as temperature or humidity.

The design of piezoelectric or inductive energy harvesters usually targets small size inertial oscillators weighting less than a few grams with resonant frequencies above $30 \mathrm{~Hz}$. Such high frequencies can be found in vibrations of machine tools, which are characterized by peak accelerations in the range $1-10 \mathrm{~m} \cdot \mathrm{s}^{-2}$ at frequencies between 70 $\mathrm{Hz}$ and $120 \mathrm{~Hz}$. For an harmonic excitation of $2.5 \mathrm{~m} . \mathrm{s}^{-2}$ at $100 \mathrm{~Hz}$, it was shown that harvesting generators designed for powering wireless acceleration sensor nodes should dissipate at least about $3 \mathrm{~mW}$ into an optimal load resistor [1]. In these working conditions, with a duty cycle of $10 \mathrm{~s}$ for transmitting data, a storage capacitor and a control unit are necessary for delivering a regulated voltage levels between 2 and $3 \mathrm{~V}$ to the circuit elements of the sensor node.

Concerning energy harvesting from bridge vibrations, a feasibility study has been carried out by Williams et al. [2], combining measured vibrational responses of small span concrete bridges with a theoretical model of energy harvester previously proposed by Williams and Yates [3]. Considering a typical recorded acceleration signal (with a maximum amplitude of $0.2 \mathrm{~m} . \mathrm{s}^{-2}$ in a frequency range below $20 \mathrm{~Hz}$ ), the authors found that an inductive generator weighting about $1 \mathrm{~kg}$ was necessary to fulfill the requirements of low power electronics. Despite significant progress in lowering the energy consumption of electronic components, similar design guidelines were more recently derived by N.G. Elvin et al. [4]. Using the theoretical solution of the moving load problem, Ali and al. [5] have recently estimated the electrical energy generated by a vehicle crossing a highway bridge. The maximum energy generated was found to be about a few microjoules. These theoretical estimates were however obtained for a small scale piezoelectric harvester with a $2.5 \mathrm{~g}$ tip mass. In the paper of Farinholt et al. [6], the use of multi-sources of ambient energy was experimentally investigated (thermal and vibration energy harvesters). Harvesting ambient bridge vibrations appeared to be most promising from the test results that were carried out with a piezo-electric generator of cantilevered beam type, $20 \mathrm{~cm}$ length. Collected acceleration data were then replayed on an electrodynamic shaker for assessing the performances of a complete harvesting system using a commercial rectifier-energy management circuit for providing a controlled voltage between $1.8 \mathrm{~V}$ and $3.6 \mathrm{~V}$ through a storage capacitor. The average power related to the charge of a $1 \mathrm{mF}$ capacitor in this voltage range was $7.5 \mu \mathrm{W}$. For comparison, an other experimental investigation in operational conditions with a $68 \mathrm{~cm}^{3}$ non resonant inductive harvester - robust to frequency mistuning - gave an estimate of the average power in the range of $0.5-0.75 \mu \mathrm{W}[7]$.

As the design of vibrational energy harvesters must target the available ambient sources, this work aims at giving a deeper insight into the traffic induced vibration 
characteristics measured on a bridge at different source locations. For the bridge example considered, the predicted performances of a validated model of piezoelectric harvesting device are subsequently discussed in relation with the traffic intensity. More precisely, the outline of this paper is as follows. In section 2 are presented some vibration measurements carried out on a highway bridge, located on a heavily trafficked itinerary. Building on the collected experimental data, a piezoelectric harvester has been designed and tested. The main results of that experimental study are collected in section 3 . In section 4 is introduced a lumped model of the piezoelectric harvester. Experimental results under harmonic excitation are used to validate that model. Section 5 is devoted to the theoretical analysis of the harvested power under traffic-induced excitations. Combining the observations of section 2 and the model of section 4 with general results from signal theory, a simple formula is obtained for estimating the harvested power in terms of traffic statistics.

\section{Traffic induced bridge vibration measurements}

\subsection{Case study}

Traffic induced vibrations have been measured on a prestressed concrete highway bridge located on a heavily trafficked itinerary in the north of France (Figure 1). A 33 m long simply supported span carrying three one way lanes has been instrumented with 4 accelerometers. The structure consists of five cross braced girders as shown in Figures 1-2(left).

From traffic data delivered by the ministry of transport, the mean flow of heavy lorries was about 8000 per day with large variations according to the day of the week and time in the day. Weigh-in-motion (WIM) data recorded in 2004 has shown that a high traffic intensity corresponds to 400 lorries per hour [8].

The work in [8] was dealing with the traffic effects on this particular bridge. It was noticed that the vehicle-bridge dynamic interaction was sensitive to the characteristics of the vehicle suspension. Negligible dynamic effects were observed on the measured strain responses for most of the moving five axles semi-trailer vehicles. For that type of lorry (which represented $80 \%$ of the heavy gross weight lorries population crossing the bridge), the weak dynamic impact factor on small span bridges was attributed to the pneumatic system in the vehicle suspension.

\subsection{Time-domain analysis of vibration measurements}

Acceleration time series were measured with a sampled frequency of $300 \mathrm{~Hz}$ on girders 1 and 3 of the simply supported span at three locations labeled as 1,2,3 in Figure 4. An example of recorded acceleration signal is displayed in Figure 5. For a high traffic intensity, the root mean square value of the measured acceleration at mid-span on girder 1 - which is more heavily loaded - was as low as $0.03 \mathrm{~m} \cdot \mathrm{s}^{-2}$. Significantly higher levels of vibration were measured on a water pipe fixed on the girder 1 (see Figure 2(right)), 


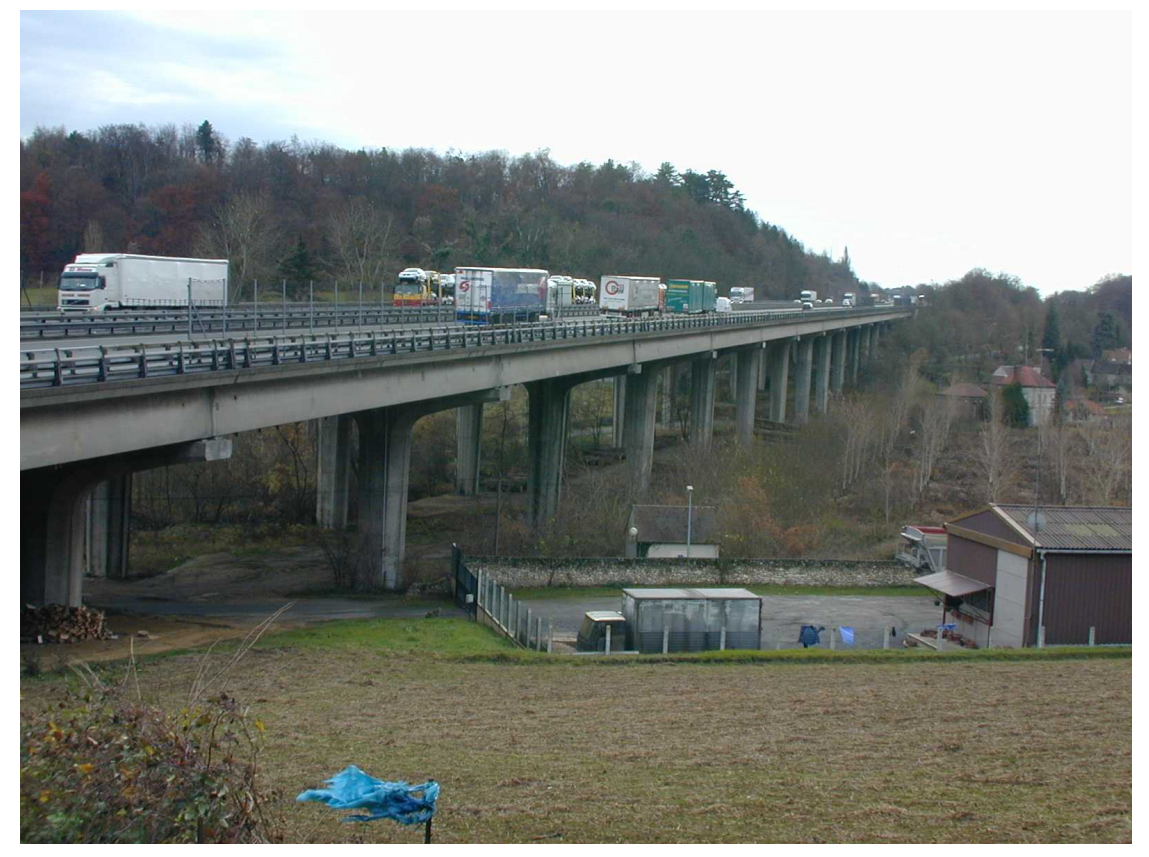

Figure 1. Bridge considered in the study.
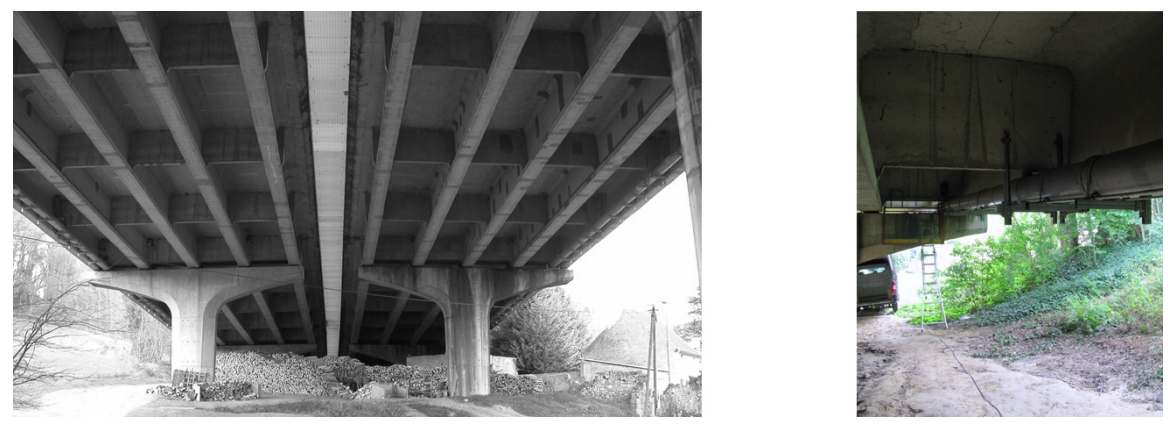

Figure 2. Bridge superstructure (left) and view of a water pipe on girder 1 (right).

especially at the location labeled as 0 in Figures 3-4. An example of acceleration time series recorded at location 0 on the pipe is displayed in Figure 5. The root mean square value of the measured acceleration for a high traffic intensity was $0.3 \mathrm{~m} . \mathrm{s}^{-2}$, ten times the estimated value on the girder. For low traffic intensity, the root mean square value of acceleration decreased to $0.1 \mathrm{~m} . \mathrm{s}^{-2}$. These estimates give the expected range of acceleration to be considered for this ambient source of vibration. The same vibration levels were measured on the water pipe fixed on girder 5 .

A closer look at the acceleration time series reveals that the signal consists of successive short-time pulses, each pulse corresponding to an individual lorry crossing the bridge. This is especially clear in night time, for which traffic intensity is low enough for an inter-arrival time to exceed the time to cross the bridge. Examples of pulses due to single lorries are shown on Figure 6. The calculated average duration of the pulse was $2 \mathrm{~s}$, which corresponds to the time for a five axles semi-trailer vehicle to cross the span of the bridge in free flowing traffic conditions. The average acceleration 


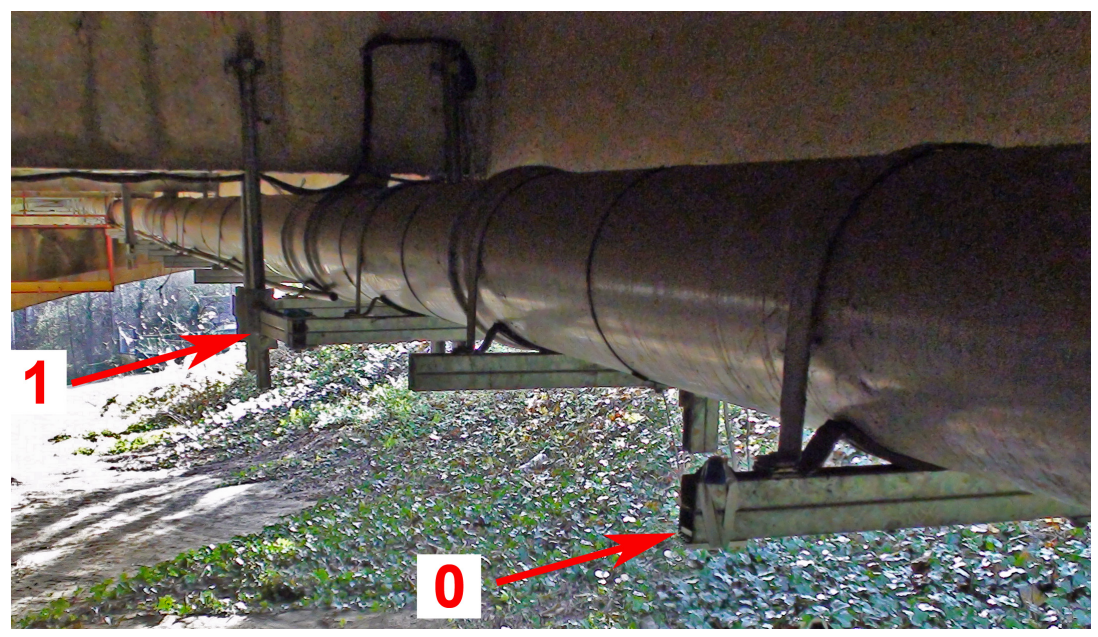

Figure 3. Locations of the sensors on the pipe.

peak level was $0.58 \mathrm{~m} . \mathrm{s}^{-2}$. In high traffic conditions, there is some overlap between the excitations due to each lorry, resulting in a signal that is more difficult to interpret (Figure 5). Light vehicles produces a low-level signal (ambient noise) with no discernible structure.

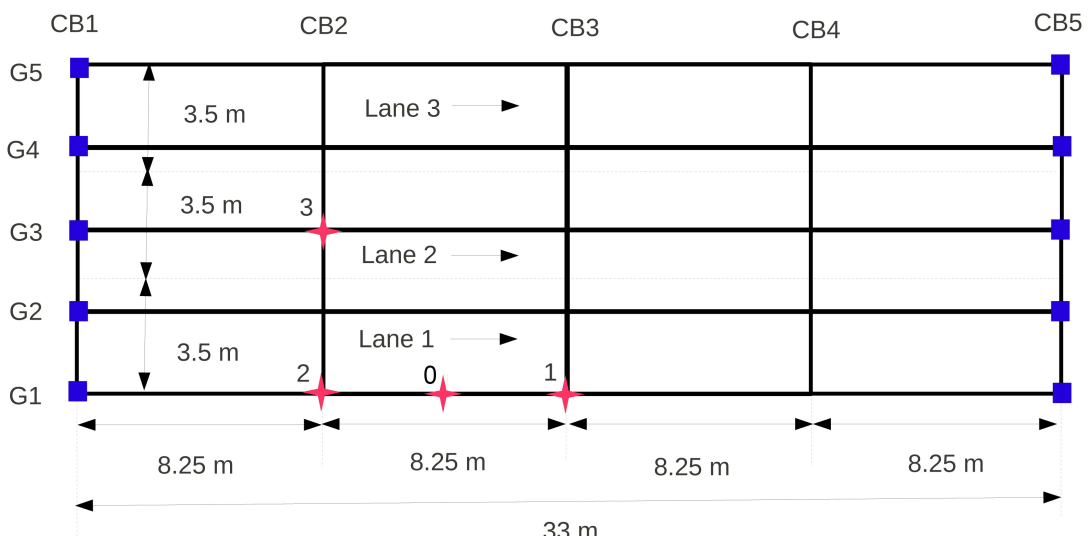

Figure 4. Scheme of bridge details.

\subsection{Frequency-domain analysis of vibration measurements}

A spectral analysis of the recorded signals was performed in order to determine the frequency content of the vibration sources on the bridge. Smoothed power spectra of the acceleration responses to the traffic excitation were estimated by averaging 360 power spectra of 3000 samples blocks. Three main resonant frequencies of the bridge deck were identified in the frequency range from 0 to $50 \mathrm{~Hz}$. Two close frequencies were in the vicinity of $4 \mathrm{~Hz}$ and an other one was found at $14.5 \mathrm{~Hz}$ (Figure 7). The results of a previous experimental modal analysis [9] carried out with a stochastic subspace 

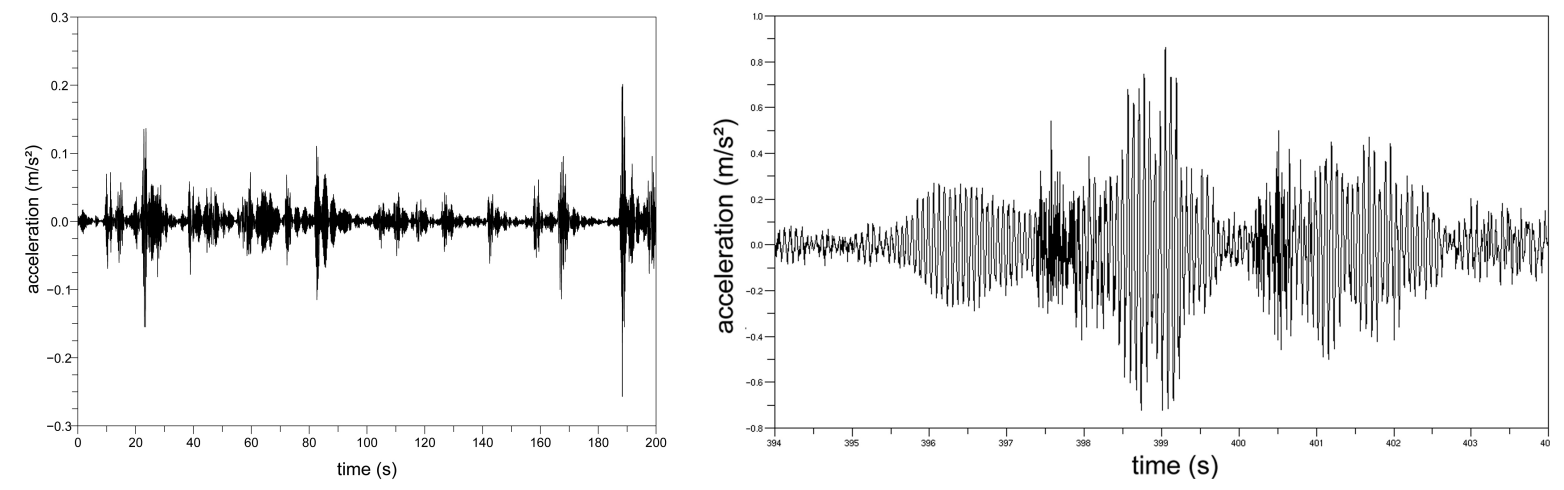

Figure 5. Recorded traffic induced acceleration measured at mid-span on girder 1 (left) and on a water pipe on girder 1 (right).
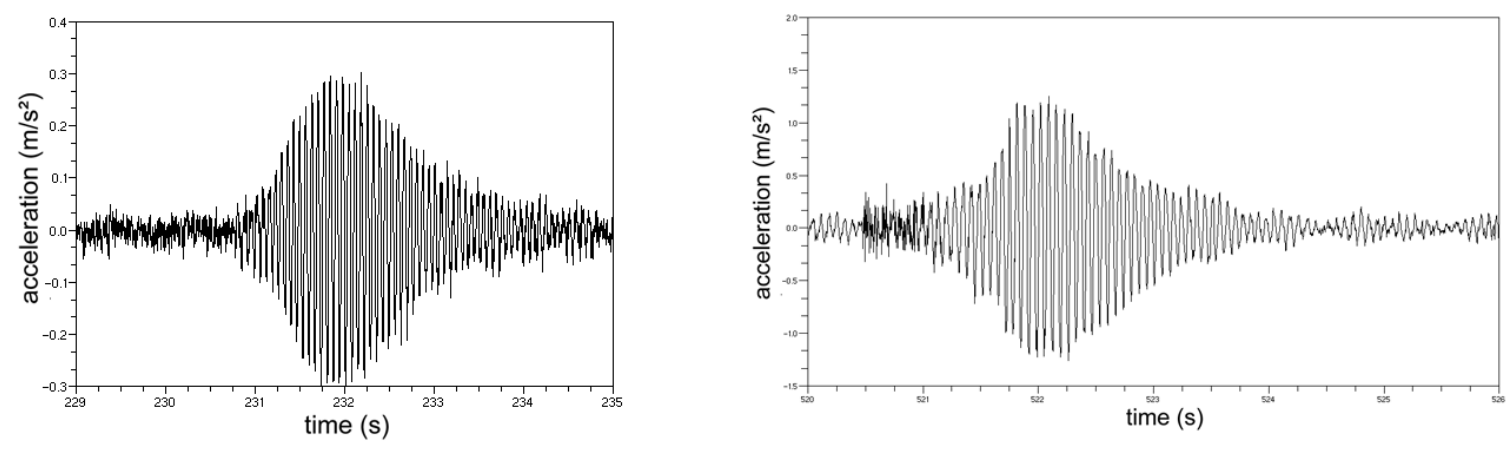

Figure 6. Examples of burst excitation due to a single lorry.

identification method (SSI) have shown that the two close frequencies were located at 3.9 $\mathrm{Hz}$ and $4.3 \mathrm{~Hz}$. These resonant frequencies are related to the mode shapes displayed in Figure 8 and correspond to the longitudinal bending, torsional and transversal bending deformations of the deck bridge. Those experimental results are consistent with the theoretical modal analysis based on a finite element model of the deck bridge [10]. Anti-nodes for all these modes shapes are located at midspan of the girders 1 and 5 . The variations of the resonant frequencies of the bridge identified from data collected during a monitoring period of 6 months are displayed in Figure 9 as a function of the ambient temperature. The uncertainty in the frequency estimates was about $0.1 \mathrm{~Hz}$ and is related to fluctuations in the traffic excitation conditions. As shown in the linear regression relationship in Figure 9, the variation of the frequency of the first bending mode induced by a $25^{\circ} \mathrm{C}$ ambient temperature change did not exceed $0.1 \mathrm{~Hz}$. However, a clear correlation relationship was not found for the others vibration modes.

To get a better understanding of the vibrations of the pipe, vibrations measurements have been done at several locations along the the pipe (labeled as 0,1,2 on Figures 3-4). The corresponding power spectra are represented in Figure 7(right). The frequency content is found to be dependent on the location considered. Both the mode shape of the bridge and the fixation details of the pipe most probably have an influence on 
the vibrations on the pipe. Comparing with the power spectra measured on the bridge (Figure 7 (left)) reveals a resonant behaviour of the pipe at frequency $14.5 \mathrm{~Hz}$.
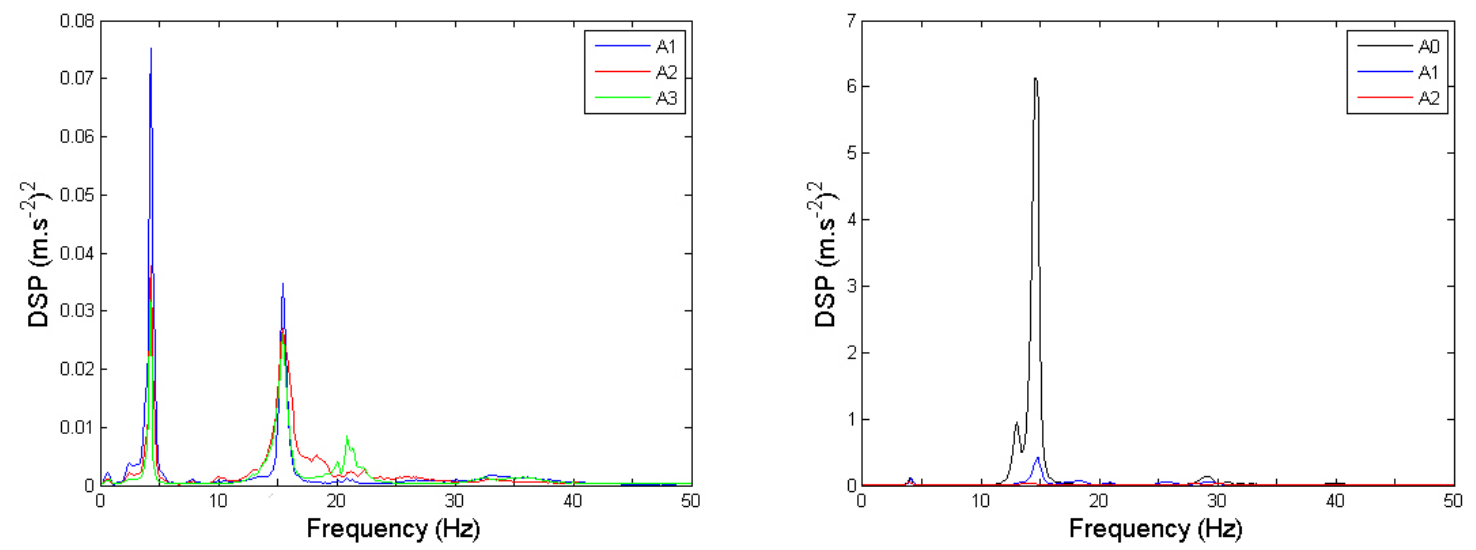

Figure 7. Power spectral densities of measured accelerations at different locations on the girders(left) and on the pipe fixed on girder 1 (right).

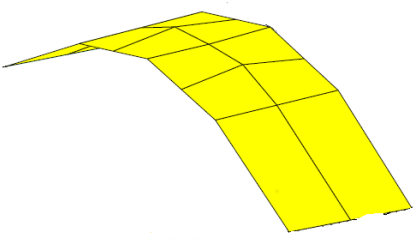

$3.9 \mathrm{~Hz}$

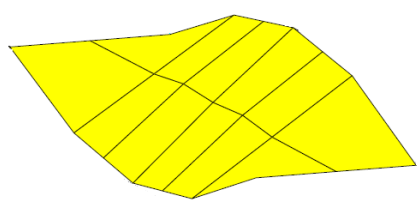

$4.3 \mathrm{~Hz}$

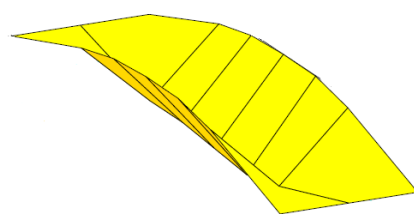

$14.5 \mathrm{~Hz}$

Figure 8. Experimental mode shapes of the bridge deck.

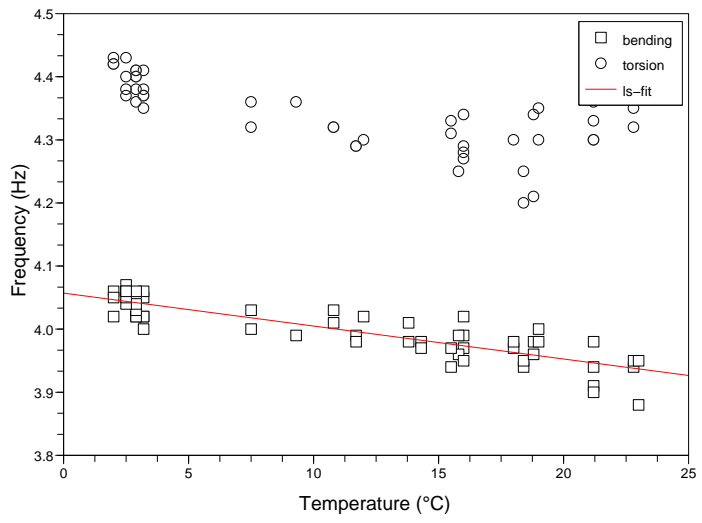

Figure 9. Resonant frequencies vs ambient temperature. 


\section{Experimental study of energy harvesting performance}

\subsection{Design of the piezoelectric harvester}

A piezoelectric energy harvesting device was designed for investigating operational conditions and comparing the measured performances with theoretical predictions. The main design requirement was to tune the resonant frequency of the inertial harvester close to $14.5 \mathrm{~Hz}$, thus targeting the resonant vibration of the pipe identified in Section 2.3. That requirement did not match the specifications of commercial energy harvesters, which are generally designed for frequencies above $30 \mathrm{~Hz}$. The piezoelectric harvester that was devised is of the cantilever type. Two bimorph piezoelectric patches Midé QP20W were bonded on the upper and lower surface of a $40 \times 220 \times 0.8 \mathrm{~mm}^{3}$ steel plate. Those bimorph piezoelectric patches were bonded at the clamped end side of the plate. An additional $12 \mathrm{~g}$ concentrated mass was placed on the steel surface for tuning the resonant frequency of the oscillator. A picture of the device is displayed in Figure 10. The material parameters provided in the manufacturer's datasheet are reported in Table $1\left(E_{p}\right.$ and $E_{e}$ are respectively the Young modulus of the piezoelectric material and of the epoxy, $\rho_{p}$ and $\rho_{e}$ are the mass densities, $e_{31}$ is the piezoelectric constant, $\epsilon_{33}^{s}$ is the relative permittivity at constant strain).
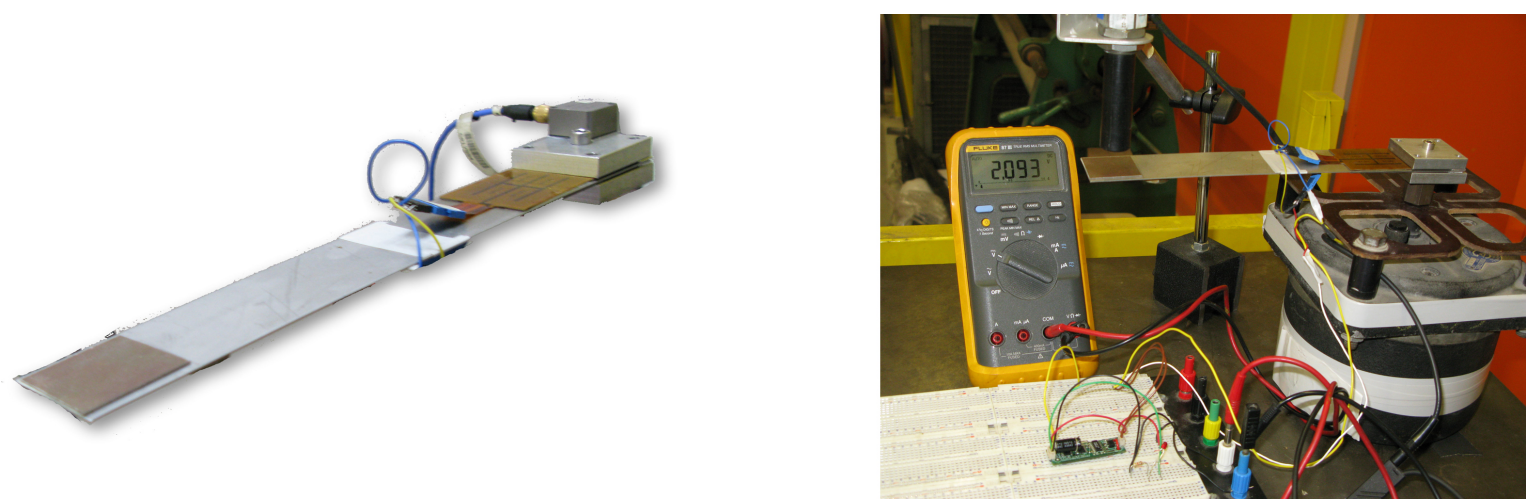

Figure 10. Piezoelectric harvester designed for traffic-induced vibrations (left), and view of the test set-up (right).

\begin{tabular}{|c|c|c|c|c|c|}
\hline$E_{p}$ & $E_{e}$ & $e_{31}$ & $\epsilon_{33}^{s}$ & $\rho_{p}$ & $\rho_{e}$ \\
\hline \multicolumn{2}{|c|}{$\mathrm{MPa}$} & $\mathrm{C} . \mathrm{m}^{-2}$ & & $\mathrm{~kg} . \mathrm{m}^{-3}$ & $\mathrm{~kg} . \mathrm{m}^{-3}$ \\
\hline 69000 & 5000 & 13.1 & 1700 & 7800 & 8000 \\
\hline
\end{tabular}

Table 1. Material parameters of the piezoelectric patch.

\subsection{Harmonic excitation}

The experimental test set-up is shown in Figure 10(right). An electromagnetic shaker was used for applying known sinusoidal base excitations to the harvester. For the 
purpose of parameter identification and for getting reference values of power conversion, tests were first carried out with a pure resistive load $R=100 \mathrm{k} \Omega$ connected to the output of the piezoelectric electrodes wired in series. In those tests, the RMS amplitude of the harmonic excitation was set to $0.2 \mathrm{~m} . \mathrm{s}^{-2}$. The frequency of the excitation ranged from 14 to $15 \mathrm{~Hz}$. For each applied frequency, the RMS voltage $V_{R M S}$ across the resistor was measured. The red dots in Figure 11 shows the power $P^{R}=V_{R M S}^{2} / R$ as a function of the applied frequency. One can check in Figure 11 that the device had a resonant frequency close to $14.4 \mathrm{~Hz}$ and the maximum measured value of power was $0.19 \mathrm{~mW}$.

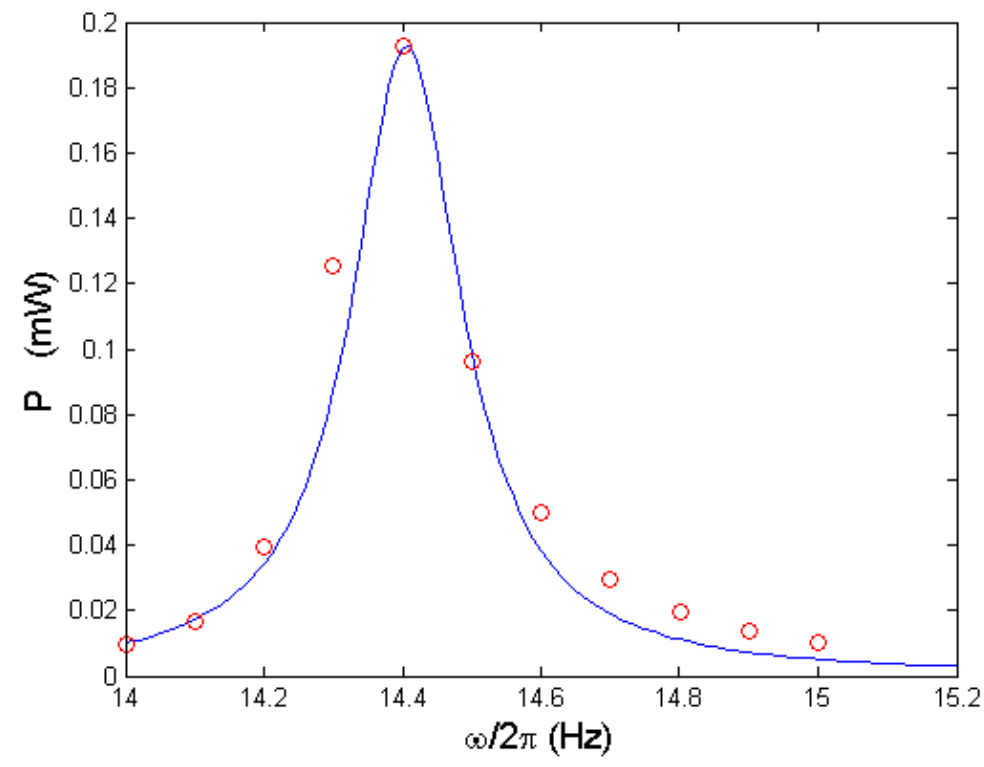

Figure 11. Harvested power for an harmonic excitation and a resistive load. Experimental measurements (red dots) and model predictions (blue solid curve).

Similar tests were subsequently performed by replacing the resistive load $R$ with an ALD EH300 circuit. That circuit provides a controlled voltage between 1.8 and $3.6 \mathrm{~V}$ through a storage capacitor, and can be used to power microelectronic devices. The energy $E^{E H}$ accumulated during one loading cycle of the capacitor is given by $E^{E H}=C^{E H}\left(V_{1}^{2}-V_{0}^{2}\right) / 2$ where $V_{0}=1.8 \mathrm{~V}, V_{1}=3.6 \mathrm{~V}$, and $C^{E H}$ is the storage capacity. The time $\Delta T$ for charging the capacitor between $1.8 \mathrm{~V}$ and $3.6 \mathrm{~V}$ in stationary harmonic regime was measured and the related mean power $P^{E H}$ was evaluated by the formula $P^{E H}=E^{E H} / \Delta T$. Figure 12 shows the harvested power $P^{E H}$ as a function of the applied frequency. The efficiency of the EH300 circuit can be assessed by comparing the results of Figure 12 with those of Figure 11: in a first approximation, the ratio $\eta=P^{E H} / P^{R}$ is constant near the resonance and equal to 0.6 . 


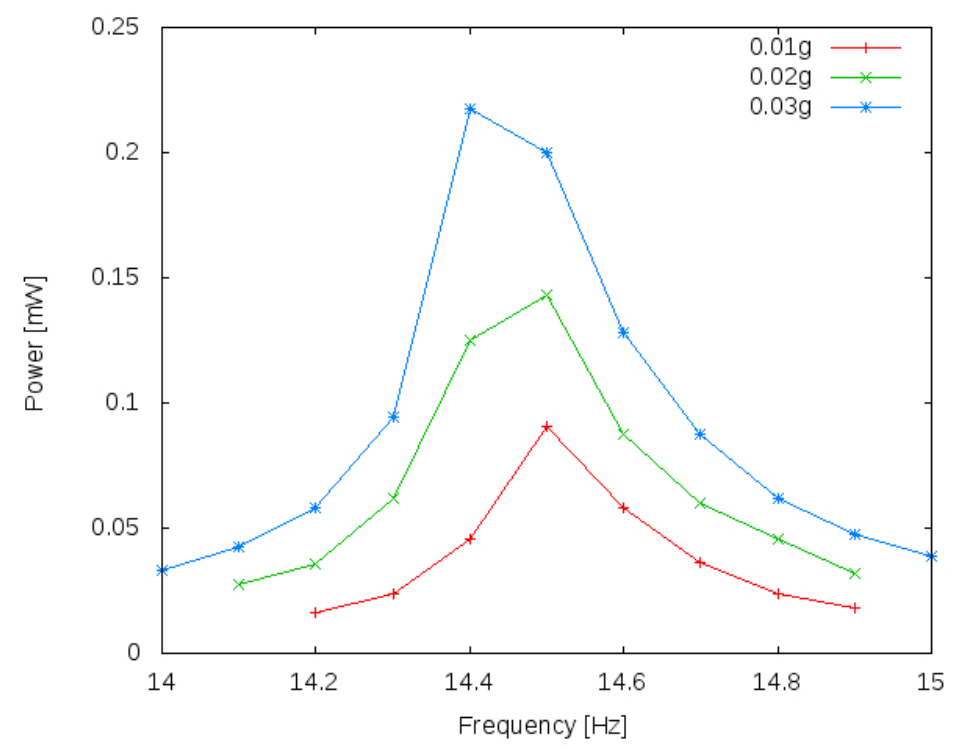

Figure 12. Harvested power using the EH300 circuit. Case of harmonic excitation.

\subsection{Traffic-induced excitation}

Further tests were carried out by replacing the harmonic excitation with the excitation measured on the pipe (at location 0 ). An example of record of the output voltage of the EH300 circuit for a real acceleration traffic excitation is shown in Figure 13. In order to check the sensitivity of the device to the frequency tuning in operational conditions, several tests were performed by slightly changing the resonant frequency of the oscillator. The results are shown as red dots in Figure 14. As could be expected, the harvested power is dramatically lower than for an harmonic excitation of the same RMS value. In good tuning conditions, the harvested power was about $0.029 \mathrm{~mW}$. It it also interesting to notice that the bandwidth of the resonance in Figure 14 is larger than in Figure 12. More precisely, as can be seen in Figures 12 - 14, the half-power bandwidth is about $0.8 \mathrm{~Hz}$ for traffic-induced excitations instead of $0.2 \mathrm{~Hz}$ for harmonic excitation. As a consequence, in the case of traffic-induced excitations, the device is less sensitive to a slight mistuning of its resonant frequency.

Some field-testing of the piezoelectric harvester was performed to complete the experimental study and check that the laboratory experiments were meaningful. The device was fixed on the pipe at location 0 shown in Figure 15(left) and connected to the EH300 circuit (see Figure 15(right)). A voltmeter was used to monitor the output of the storage capacity in the EH300 circuit. The behaviour of the storage capacity voltage was qualitatively similar to the laboratory results shown in Figure 13. The average time for fully charging the capacity was $210 \mathrm{~s}$, which corresponds to an average power of approximatively $0.024 \mathrm{~mW}$. That test notably shows that the vibrations of the pipe are not highly damped by the piezoelectric harvester, which can be attributed to the relative light weight of the harvester compared to the pipe. 


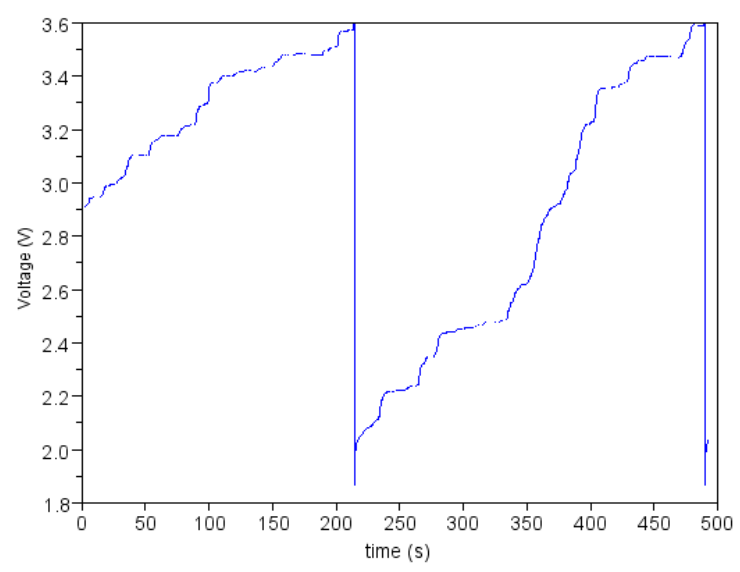

Figure 13. Storage capacity output voltage in the case of traffic induced excitation.

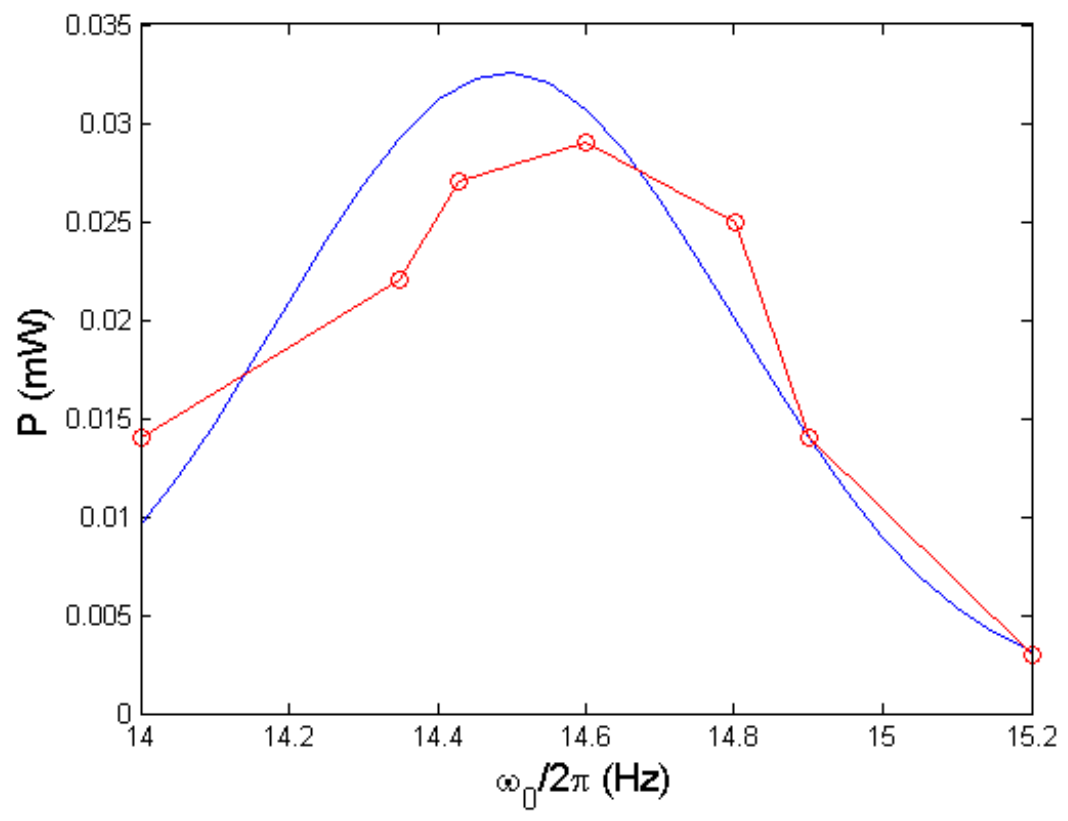

Figure 14. Harvested power in the case of traffic induced excitation, as a function of the resonant frequency. Experimental measurements (in red) and model predictions (in blue, see section 5.2).

\section{Modelling of the cantilevered piezoelectric harvester}

\subsection{Beam model}

The electromechanical modelling of cantilevered piezoelectric harvesters has extensively been studied in the literature [11]. Here we consider a single mode approximation of a Euler-Bernoulli model of the beam (see Figure 16(left)). The bending stiffness EI of the composite section made of piezoelectric patches and the steel substrate, shown in 

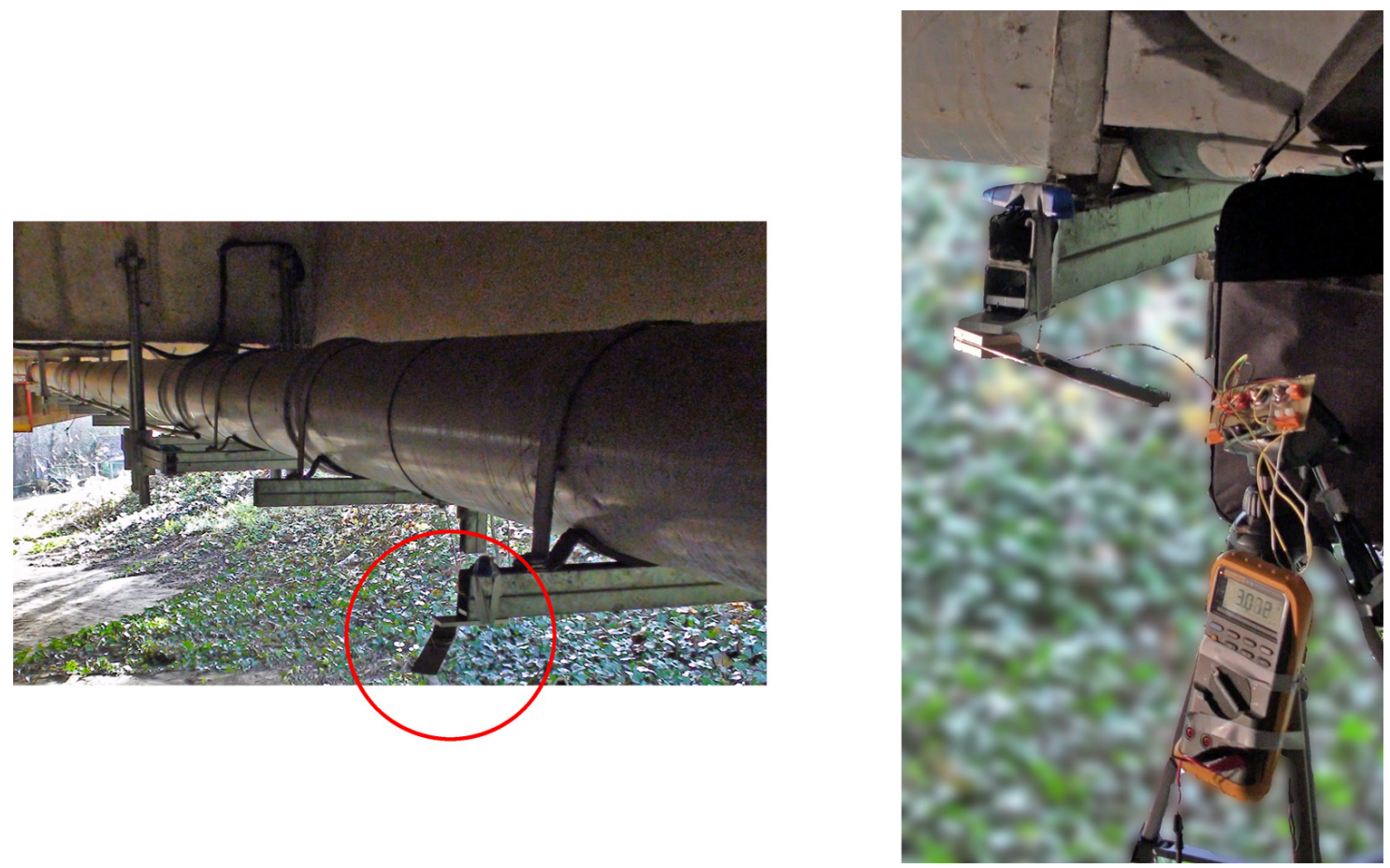

Figure 15. (left) View of the waterpipe equiped with the piezoelectric harvester, shown in the red circle. (right) Close-up view of the piezoelectric harvester, fixed on the pipe and connected to the EH300 circuit. A voltmeter is used to monitor the voltage at the output of the EH300 circuit.

Figure 16(right), is calculated assuming that the cross sections remain plane and normal to the center line of the beam. The axis $x_{1}$ is taken along the beam axis and the axis $x_{3}$ is normal to the bending plane. The lengths $h, W_{p}, W_{s}$ indicated on Figure 16(right) are given by $h=3 \mathrm{~mm}, W_{p}=33 \mathrm{~mm}, W_{e}=38 \mathrm{~mm}$.

As the piezoelectric patches did not cover the entire length of the beam, the bending stiffness was not constant along the beam. This motivated the use of a finite-element model (done with the FEM code CAST3M [12]) for calculating the modal frequencies and mode shapes of the beam. The first modal frequency $\omega_{0} / 2 \pi$ was found to be about 14.4 Hz. The corresponding mode shape $\phi\left(x_{1}\right)$ is represented in Figure 17.

The single mode approximation of the energy harvester powering a resistive load $R$ leads to the dynamic system model [13]:

$$
\begin{aligned}
& m \ddot{z}+c \dot{z}+k z-\theta v=m^{*} f \\
& \theta \dot{z}+C_{p} \dot{v}+\frac{v}{R}=0
\end{aligned}
$$

where $z(t)$ is the displacement at the tip of the beam, $v(t)$ is the voltage in the resistor, and $f(t)$ is the applied excitation. The mechanical parameters $m, m^{*}$ and $k$ 

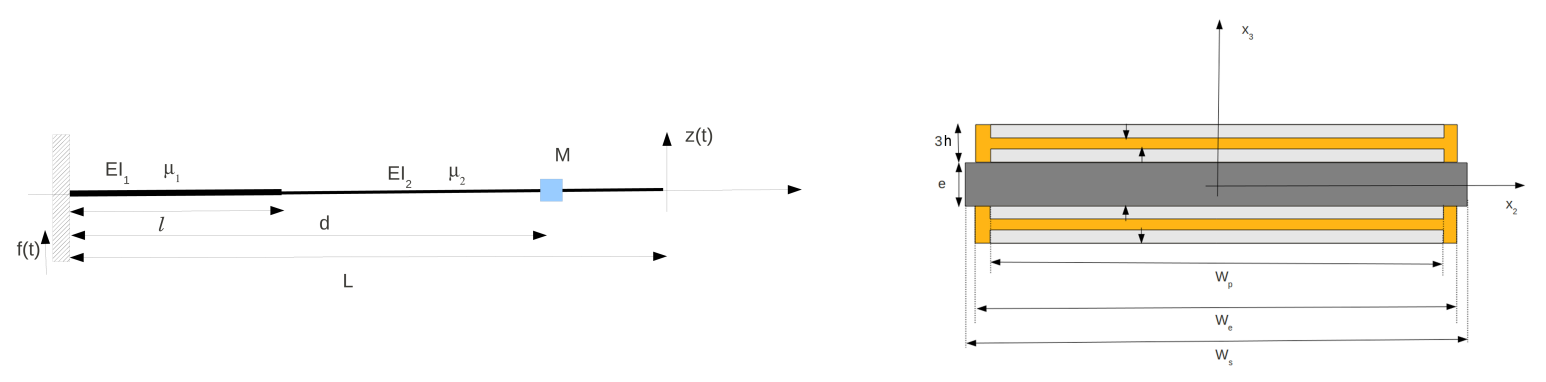

Figure 16. Geometry of the cantilevered beam (left) and composite section of beam equipped with bimorph piezoelectric patches (right).

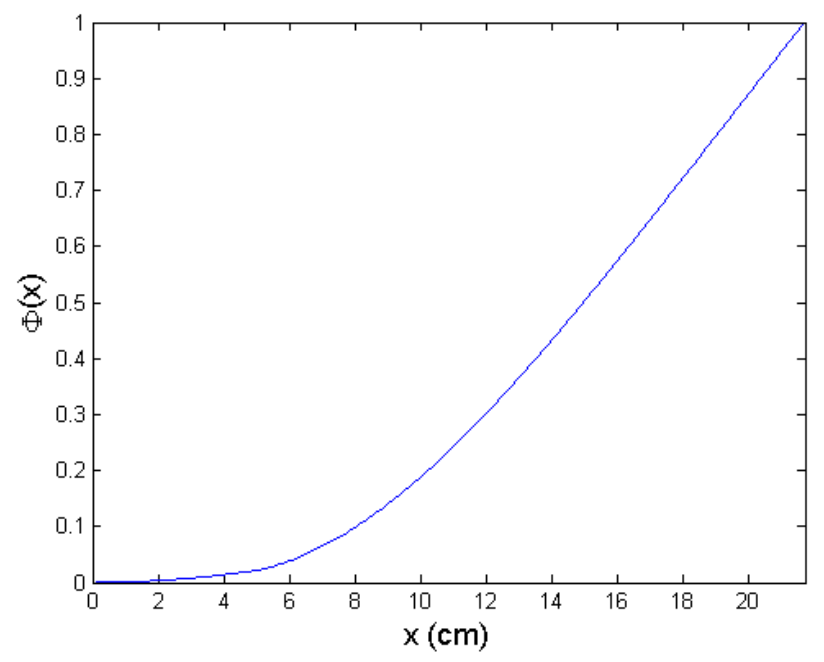

Figure 17. First mode shape for the cantilever beam.

are obtained from the mode shape $\phi\left(x_{1}\right)$ by the formulae

$$
\begin{aligned}
& m=\int_{0}^{L} \mu\left(x_{1}\right) \phi^{2}\left(x_{1}\right) \mathrm{d} x_{1} \\
& m^{*}=\int_{0}^{L} \mu\left(x_{1}\right) \phi\left(x_{1}\right) \mathrm{d} x_{1} \\
& k=\int_{0}^{L} E I\left(x_{1}\right) \phi^{\prime 2}\left(x_{1}\right) \mathrm{d} x_{1}
\end{aligned}
$$

where $\mu\left(x_{1}\right)$ and $E I\left(x_{1}\right)$ are respectively the linear mass distribution and the bending stiffness distribution along the beam model. In the above relations, the mode shape $\phi$ is assumed to be normalised with respect to the degree of freedom $z$ considered for the displacement. The parameter $c$ is a viscous damping coefficient that accounts for energy dissipation in the mechanical oscillator.

In (1), the expressions of the piezoelectric coupling term $\theta$ and of the capacity $C_{p}$ depend on the poling directions in each piezoelectric layer as well as on the connections 
of the piezoelectric electrodes (series or parallel). The formulae for series electrical wiring are

$$
\begin{aligned}
& \theta=e_{31} W_{p} \frac{e+3 h}{2} \phi^{\prime}(l) \\
& C_{p}=\epsilon_{0} \epsilon_{33}^{s} \frac{l W_{p}}{4 h}
\end{aligned}
$$

The expression (4) corresponds to the capacity of a series association of 4 parallel plate condensers with capacity $\epsilon_{0} \epsilon_{33}^{s} l W_{p} / h$. More details on the derivation of $\theta$ are given in the Appendix.

\subsection{Parameter identification and model validation}

With the exception of the linear damping coefficient $c$, the values of all the system parameters in (1) are determined by the relations (2)-(4) and the material parameters in Table 1 . The viscous damping coefficient $c$ was estimated from free decay vibrations in open circuit conditions. We found a mean value of $c$ equals to 0.02 N.s.m ${ }^{-1}$. The values of all the system parameters are reported in Table 2.

The measured mean electrical power displayed in Figure 11 is now compared with the prediction of the theoretical model, using the values in Table 2. For an harmonic acceleration $f(t)=\operatorname{Re}\left(F e^{i \omega t}\right)$, the displacement $z(t)$ and voltage $v(t)$ solutions of (1) take the form

$$
z(t)=\operatorname{Re}\left(Z e^{i \omega t}\right), v(t)=\operatorname{Re}\left(V e^{i \omega t}\right)
$$

where $Z$ and $V$ satisfy

$$
\begin{array}{ll}
-m \omega^{2} Z+i \omega c Z+k Z-\theta V & =m F \\
i \omega \theta Z+i C_{p} \omega V+\frac{v}{R} & =0
\end{array}
$$

Solving that system shows that $V$ can be written as

$$
V=\frac{F}{H(\omega)}
$$

where

$$
H(\omega)=\frac{1}{\theta}\left(-C_{p}\left(\omega_{0}^{2}-\omega^{2}\right)-\frac{\nu}{R}\right)-\frac{\theta}{m}+\frac{i}{\theta}\left(\frac{\omega_{0}^{2}-\omega^{2}}{R \omega}-\omega \nu C_{p}\right)
$$

In that equation, $\nu$ is defined by $\nu=c / m$. Let $P$ denote the average power (per cycle) delivered to the load resistance. We have

$$
P=\frac{1}{2 R} \frac{|F|^{2}}{|H(\omega)|^{2}}
$$

The theoretical power is represented as a blue curve on Figure 11 and is found to provide good agreement with the measurements. The peak power is about $0.2 \mathrm{~mW}$ and the half-power bandwidth is approximatively equal to $0.2 \mathrm{~Hz}$. 
Piezoelectric energy harvesting from traffic-induced bridge vibrations

\begin{tabular}{|c|c|c|c|c|c|}
\hline $\begin{array}{c}m \\
(\mathrm{~g})\end{array}$ & $\begin{array}{c}k \\
\left(\mathrm{~N} . \mathrm{m}^{-1}\right)\end{array}$ & $\begin{array}{c}c \\
\left(\mathrm{~N} . \mathrm{s} \cdot \mathrm{m}^{-1}\right)\end{array}$ & $\begin{array}{c}\theta \\
\left(\mathrm{N}^{-} \mathrm{V}^{-1}\right)\end{array}$ & $\begin{array}{c}m^{*} \\
(\mathrm{~g})\end{array}$ & $\begin{array}{c}C_{p} \\
(\mathrm{nF})\end{array}$ \\
\hline 23 & 188 & 0.02 & $2.3410^{-4}$ & 21.4 & 2.24 \\
\hline
\end{tabular}

Table 2. Lumped model parameters.

\section{Analysis and prediction of power conversion}

\subsection{Maximum power conversion}

For a given acceleration amplitude $F$, the power $P$ in (8) depends on the load resistance $R$ and on the excitation frequency $\omega$. Maximizing $P$ with respect to those two parameters leads to solve the equation $\partial P / \partial R=0$ and $\partial P / \partial \omega=0$. The equation $\partial P / \partial R=0$ gives the optimal resistance at a fixed $\omega$ and can be rewritten as

$$
|H(\omega)|^{2}+R \frac{\partial|H(\omega)|^{2}}{\partial R}=0
$$

That equation is found to translate as a second degree polynomial in $1 / R$. The solution is

$$
R(\omega)=\sqrt{\frac{\nu^{2}+\left(\omega_{0}^{2}-\omega^{2}\right)^{2} / \omega^{2}}{\left(C_{p}\left(\omega_{0}^{2}-\omega^{2}\right)+\theta^{2} / m\right)^{2}+C_{p}^{2} \omega^{2} \nu^{2}}}
$$

The equation $\partial P / \partial \omega=0$ gives the optimal pulsation at a given load resistance $R$ and is equivalent to

$$
\frac{\partial|H(\omega)|^{2}}{\partial \omega}=0
$$

That equation translates as

$$
2 C_{p}\left(\frac{\theta}{m}+\frac{1}{\theta}\left(C_{p}\left(\omega_{0}^{2}-\omega^{2}\right)+\frac{\nu}{R}\right)\right)+\frac{1}{\theta}\left(\frac{\omega_{0}^{4}}{R^{2} \omega^{4}}-\left(\frac{1}{R}+\nu C_{p}\right)^{2}\right)=0
$$

which is a $6^{\text {th }}$ degree polynomial in $\omega$. One is particularly interested is the case where the systems of equations (9-10) are solved together with respect to $(\omega, R)$. The corresponding solution, denoted by $\left(\omega_{*}, R_{*}\right)$, is the optimal operating point of the circuit, i.e. the set of parameters that maximizes the electrical power. Using the expression (9$10), \omega_{*}$ is found to be solution of an equation of the form

$$
A(\omega) C^{\prime}(\omega)+A^{\prime}(\omega) C(\omega)=0
$$

with

$$
\begin{aligned}
& A(\omega)=\nu^{2}+\left(\frac{\omega_{0}^{2}-\omega^{2}}{\omega}\right)^{2} \\
& C(\omega)=\left(C_{p}\left(\omega_{0}^{2}-\omega^{2}\right)+\frac{\theta^{2}}{m}\right)^{2}+C_{p}^{2} \omega^{2} \nu^{2} \\
& A^{\prime}(\omega)=2 \omega\left(1-\frac{\omega_{0}^{4}}{\omega^{4}}\right) \\
& C^{\prime}(\omega)=2 C_{p} \omega\left(C_{p} \nu^{2}-2\left(C_{p}\left(\omega_{0}^{2}-\omega^{2}\right)+\frac{\theta^{2}}{m}\right)\right)
\end{aligned}
$$

That equation is a $6^{\text {th }}$ degree polynomial equation in $\omega$ and does not have any obvious solution. An assumption frequently used in literature is to consider that $\left.\omega_{0}=\sqrt{(} \mathrm{k} / \mathrm{m}\right)$ 
is solution of (11). It is clear from (11-12) that this assumption is true only in the degenerate case $\nu=0$. Assume, however, that (11) admits a solution $\omega_{*}$ close to $\omega_{0}$. Using a first-order expansion of $\omega_{*}$ in the form $\omega_{*}=\omega_{0}+d \omega$ (with $|d \omega| \ll \omega_{0}$ ) we obtain

$$
\omega_{*}=\omega_{0}+X \omega_{0}
$$

where $X$ is the dimensionless scalar defined as

$$
X=\frac{-C_{p} \nu^{2}\left(C_{p} \nu^{2}-2 \theta^{2} / m\right)}{\left(C_{p} \nu^{2}-2 \theta^{2} / m\right)^{2}+8 \omega_{0}^{2} C_{p}^{2} \nu^{2}+2 C_{p} \nu^{2} \theta^{2} / m}
$$

That first-order expansion is justified if the system parameters are such that $X \ll 1$. In that case, the assumption $\omega_{*}=\omega_{0}$ can be interpreted as a 0th-order expansion of the solution of (11). A more precise first-order expansion is given by (13-14). For the constitutive parameters in Table 2 , we obtain that $X \simeq 3.510^{-4}$, so that the estimation $\omega_{*} \simeq \omega_{0}$ is indeed a good approximation in our case. The corresponding optimal power (for an input acceleration of amplitude $0.2 \mathrm{~m} . \mathrm{s}^{-2}$ ) is equal to $0.226 \mathrm{~mW}$. That value is reached for the optimal resistance $R_{*}=R\left(\omega_{*}\right)=364 \mathrm{k} \Omega$.

In real applications, the input acceleration has little chance to be tuned to the optimal frequency. It does not even have to be harmonic, nor periodic. More over, the impedance of electronic circuits powered by the harvester doe not necessarily match the optimality conditions. In fact, such circuits often involve rectifying circuits and have a nonlinear behaviour, exhibiting threshold effects. Because of all those effects, the formula (8) is only expect to deliver an upper bound on the energy that is harvested in practice. Deviation with respect to that upper bound are investigated in the following.

\subsection{Prediction of power conversion for traffic-induced excitations}

As presented in section 2.2, traffic-induced excitations consist of sequences of shorttime pulses, corresponding to individual lorries passing by. There is obviously some fluctuation in the detailed structures of those pulses, as well as in the delay between them. As detailed in section 2.2, an individual pulse is a modulated periodic signal with an inner frequency $\omega_{i}$ related to the modal frequencies of the bridge and the location of the harvester. In this section, a pulse is modelled as a function of the form

$$
A f_{0}(t)
$$

where

$$
f_{0}(t)=\operatorname{tri}(t) \sin \omega_{i} t
$$

and $\operatorname{tri}(t)$ is the triangle function defined as

$$
\operatorname{tri}(t)= \begin{cases}0 & \text { for }|t|>M \\ 1+t / M & \text { for }-M \leq t \leq 0 \\ 1-t / M & \text { for } 0 \leq t \leq M\end{cases}
$$

That model is compatible with the experimental observations of section 2.2, and mathematically simple enough for the most of the subsequent analysis to be performed 


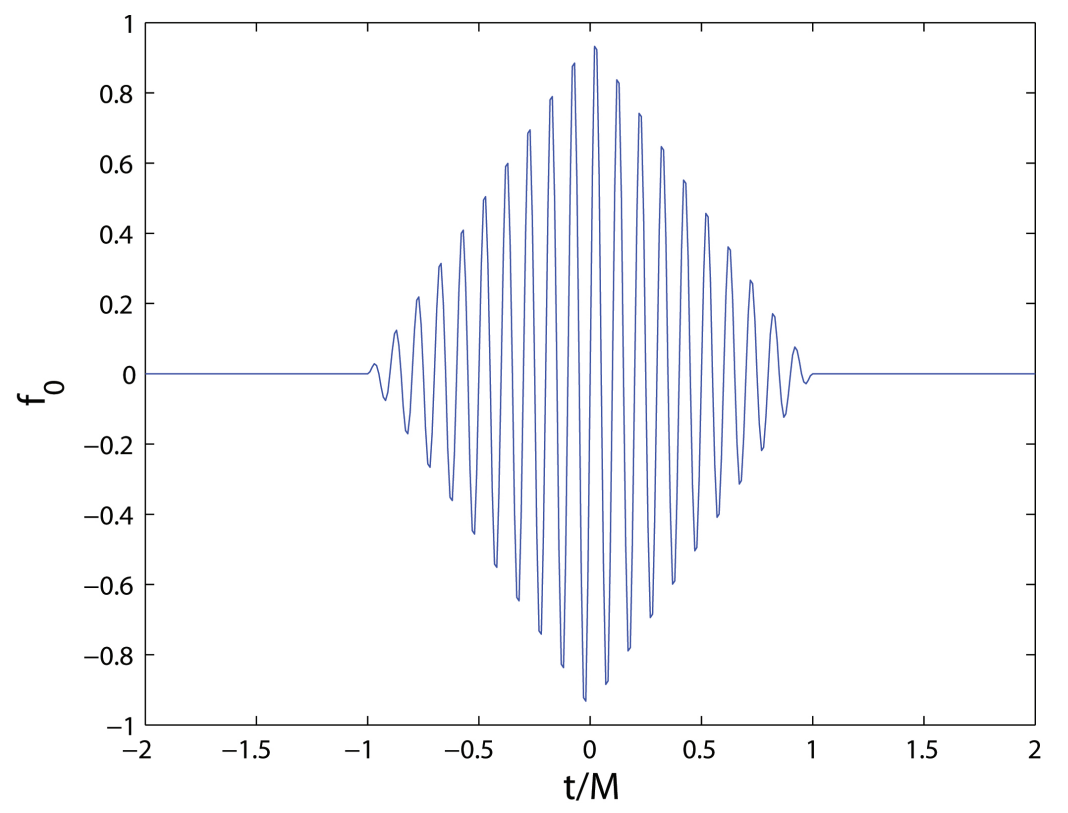

Figure 18. Model of a pulse excitation.

in closed-form. The function $f_{0}$ is represented on Figure 18. In (15), the parameter $M$ characterizes the duration of the pulse and $A$ is the peak level.

A simple calculation shows that the Fourier transform of $f_{0}$ is

$$
\hat{f}_{0}(\omega)=\frac{M}{2}\left(e^{-i M\left(\omega_{i}-\omega\right)} \operatorname{sinc}^{2} \frac{M\left(\omega_{i}-\omega\right)}{2}+e^{-i M\left(\omega_{i}+\omega\right)} \operatorname{sinc}^{2} \frac{M\left(\omega_{i}+\omega\right)}{2}\right)
$$

It follows that

$$
\left|\hat{f}_{0}(\omega)\right|^{2}=\frac{M^{2}}{4}\left(\operatorname{sinc}^{4} x+\operatorname{sinc}^{4} y+2 \cos (M \omega) \operatorname{sinc}^{2} x \operatorname{sinc}^{2} y\right)
$$

where $x=M\left(\omega_{i}+\omega\right) / 2$ and $y=M\left(\omega_{i}-\omega\right) / 2$.

5.2.1. Periodic train of pulses Let us first consider a periodic excitation $f$ constituted of pulses of type (15) occurring at frequency $N_{f}$. The Fourier series of the function $f$ is

$$
\hat{f}_{k}=A N_{f} \hat{f}_{0}\left(2 \pi k N_{f}\right)
$$

Using the constitutive equations presented in section 4 , the average power $P^{R}$ harvested in a purely resistive load $R$ is given by

$$
P^{R}=\frac{A^{2}}{R} \sum_{k \in \mathbb{Z}} \frac{\left|\hat{f}_{k}\right|^{2}}{\left|H\left(2 \pi k N_{f}\right)\right|^{2}}
$$

The curves on Figure 19 shows the power given by (18) as a function of $N_{f}$, using the values in Table 2 for the constitutive parameters. In accordance with the results of 
section 2.2 , the frequency $\omega_{i} / 2 \pi$ is set equal to $14.5 \mathrm{~Hz}$ and the parameters $(A, M)$ are set equal to $A=0.58 \mathrm{~m} . \mathrm{s}^{-2}, M=1 \mathrm{~s}$. On Figure 19 clearly appear two regimes, separated by a characteristic frequency $N^{*} \simeq 0.1 \mathrm{~Hz}$. For $N_{f}<N^{*}$, the harvester power $P^{R}$ is linear with respect to the frequency $N_{f}$. In that regime, use of the formula (18) gives

$$
P^{R}=N_{f} A^{2} E_{0}
$$

where

$$
E_{0}=\frac{1}{2 \pi R} \int_{-\infty}^{+\infty} \frac{\left|\hat{f}_{0}(\omega)\right|^{2}}{|H(\omega)|^{2}} d \omega
$$

In contrast, for $N_{f}>N^{*}$, the harvested power $P^{R}$ exhibits a high sensitivity with respect to $\omega_{f}$. The interpretation is that for $N_{f}<N^{*}$, the delay between two consecutive pulses is high enough for them to be essentially decorrelated, in the sense that the system has enough time to almost recover its equilibrium position after each pulse. In such condition, the harvested power is equal to the energy $E_{0}$ harvested during a single pulses multiplied by the frequency $N_{f}$ of the pulses. The behaviour of the system is more complex in the regime $N_{f}>N^{*}$ : in that range of frequencies, resonances and antiresonances phenomena appear. In particular, it can be verified that the peaks of $P^{R}$ corresponds to frequencies $N_{f}$ which are multiple of $\omega_{i} / 2 \pi$.

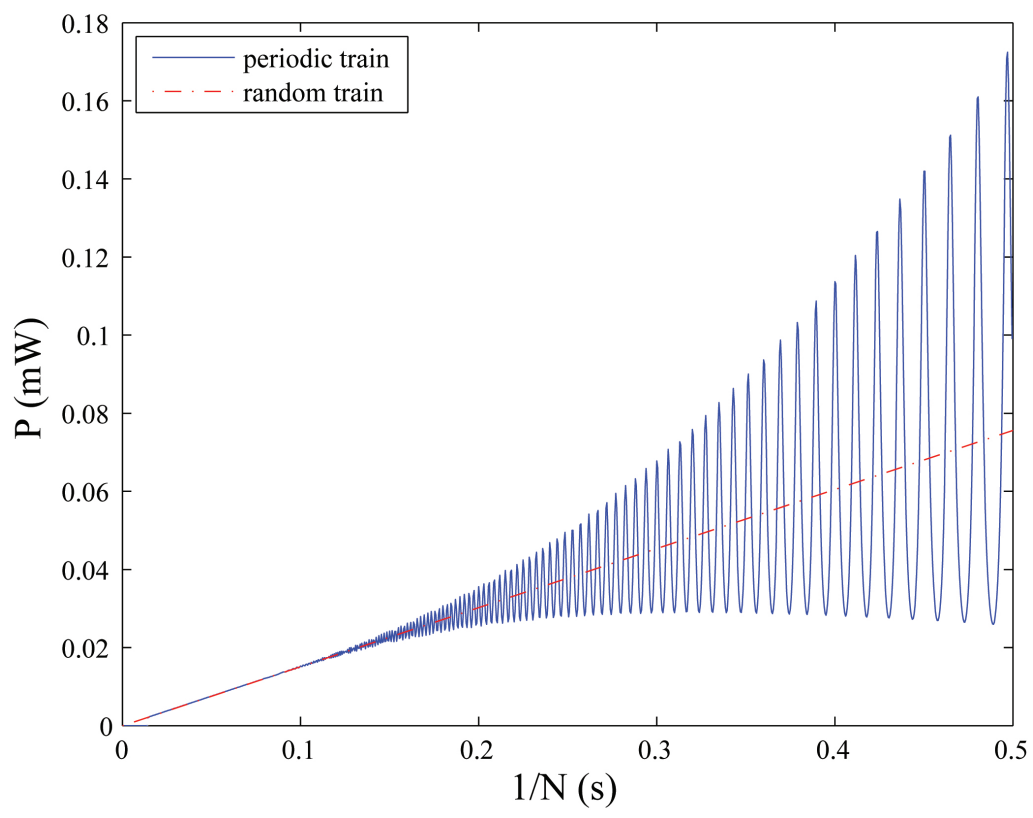

Figure 19. Prediction of the harvested power for an non-harmonic input.

5.2.2. Random train of pulses We now consider an acceleration signal $f(t)$ of the form

$$
f(t)=\sum_{k=-\infty}^{+\infty} A_{k} f_{0}\left(t-t_{k}\right)
$$


For such a non periodic excitation, the harvester power $P^{R}$ is obtained by calculating the average power on the time interval $[-T / 2, T / 2]$ and taking the limit $T \rightarrow+\infty$. More precisely, for any $T>0$ we consider the truncated signal $f_{T}(t)$ defined as $f_{T}(t)=f(t)$ if $|t| \leq T / 2$, and $f_{T}(t)=0$ otherwise. Let $V_{T}$ be the solution of (1) for the input acceleration $f_{T}$. The Fourier transform $\hat{V}_{T}$ of $V_{T}$ is given by

$$
\hat{V}_{T}(\omega)=\frac{\hat{x}_{T}(\omega)}{H(\omega)}
$$

where $H(\omega)$ is defined in (7). The average harvested power $P^{R}$ is given by

$$
P^{R}=\lim _{T \rightarrow+\infty} \frac{1}{T} \int_{-\infty}^{\infty} \frac{V_{T}^{2}(t)}{R} d t
$$

Combining Parseval theorem with (22), we obtain

$$
P^{R}=\lim _{T \rightarrow+\infty} \frac{1}{2 \pi T} \int_{-\infty}^{\infty} \frac{\left|\hat{f}_{T}(\omega)\right|^{2}}{|H(\omega)|^{2}} d \omega
$$

Let us now assume that $A_{k}$ and $t_{k}$ in $(21)$ are random variables. We use brackets \langle\rangle to denote ensemble averages. From (24) we obtain

$$
\left\langle P^{R}\right\rangle=\frac{1}{2 \pi} \int_{-\infty}^{\infty} \frac{S_{f}(\omega)}{|H(\omega)|^{2}} d \omega
$$

where $S_{f}$ is the power spectral density of $f$, defined as

$$
S_{f}(\omega)=\lim _{T \rightarrow+\infty} \frac{\left\langle\left|\hat{f}_{T}(\omega)\right|^{2}\right\rangle}{T}
$$

We refer to the textbooks of Drake [14] and Mix [15] for more details on random signal theory. For a function $f$ of the form (21) and if the random variables $A_{k}$ are uncorrelated, Carson's theorem $[15,16]$ gives

$$
S_{f}(\omega)=\nu\left\langle A^{2}\right\rangle\left|\hat{f}_{0}(\omega)\right|^{2}+2 \pi \delta(\omega)\left[\nu\langle A\rangle \int_{\infty}^{+\infty} f_{0}(t) d t\right]
$$

where $N$ is the average rate of pulse emission. Assuming that $\langle A\rangle=0$, we get

$$
\left\langle P^{R}\right\rangle=\frac{1}{2 \pi} N\left\langle A^{2}\right\rangle \int_{-\infty}^{\infty} \frac{\left|\hat{f}_{0}(\omega)\right|^{2}}{|H(\omega)|^{2}} d \omega
$$

which using (20) can be rewritten as

$$
\left\langle P^{R}\right\rangle=N\left\langle A^{2}\right\rangle E_{0}
$$

Let us now apply this knowledge to study harvesting performances under traffic induced excitations.

In night-time, the experimental measurements show that the average delay between two lorries is $18 \mathrm{~s}$. Using that value in (29) gives a power of $10.1 \mu \mathrm{W}$. In order to check that estimate, we have numerically performed a Fast Fourier Transform (FFT) of the acceleration signal and substituted the result in (18) to obtain the power. That procedure yields a power of $13.5 \mu \mathrm{W}$, which is of the same order of magnitude as the result of (29) . 
In day time, the average delay between two trucks is measured to be $3.6 \mathrm{~s}$. Calculating the power via a FFT of the day-time acceleration signal leads to a power of $59.8 \mu \mathrm{W}$, roughly five times bigger than in night time conditions. Using the formula (29) gives a power of $50 \mu \mathrm{W}$, which again is of the same order of magnitude as the value obtained by FFT of the full signal.

All the considerations so far apply to a purely resistive load $R$. If we now consider that a EH300 circuit is used, then, following the developments of section 3.2, the harvested power $P^{E H}$ can be estimated as $P^{E H}=\eta P^{R}$ where $\eta$ characterizes the relative efficiency of the circuit compared to the reference resistance $R$. The efficiency $\eta$ has been assessed in section 3.2 , leading to $\eta=0.6$ for $R=100 \mathrm{k} \Omega$. Using the formula (29), we end up with the following estimate:

$$
\left\langle P^{E H}\right\rangle=\eta N\left\langle A^{2}\right\rangle E_{0}
$$

The blue curve in Figure 14 shows the power given by (30) as a function of the resonant frequency $\omega_{0}$. That curve is to be compared with the experimental measurements (shown in red) of Figure 14, showing good agreement. In particular, the simplified formula (30) gives a good estimate of the maximum harvested power, which is close to $0.03 \mathrm{~mW}$. The formula (30) also captures the increased bandwidth compared to the case of harmonic excitations: the half-power bandwidth predicted by the model in Figure 14 is indeed approximatively equal to $0.8 \mathrm{~Hz}$, as for the experimental measurements.

\section{Conclusion}

The study of in situ measurements shows that traffic-induced vibrations in bridges consist in random train of short-time pulses. The detailed structure of those pulses notably depends on the location considered and on the modal response of the bridge. The cantilever piezoelectric harvester that has been presented in this paper targets a transverse bending mode of the bridge, corresponding to a frequency of $14.5 \mathrm{~Hz}$. For peak traffic intensity, power up to $0.03 \mathrm{~mW}$ can be produced, with a controlled voltage between 1.8 and $3.6 \mathrm{~V}$. That energy supply could be used to power wireless health monitoring sensor nodes with low cycle duty. A simple model for piezoelectric harvester under non-periodic and random excitations has been studied, leading to a simple formula relating the harvested power to traffic statistics and to the constitutive parameters of the piezoelectric device. That formula is found to agree relatively well with experimental measurements under operating conditions, and could be used as a design rule for piezoelectric harvesters fitted to bridges. In that regard, we note that the presented piezoelectric device is not necessarily optimal: more advanced design could possibly result in a better energy production / device volume ratio.

\section{Acknowledgements}

The authors are grateful to the SANEF authorities of the tested bridge for the provided support. The authors wish to thank Jean-Luc Bachelier and René-Stéphane Morelle for 
their help in setting up the experiments. The contribution of Erick Merliot to the finite element analysis is also gratefully acknowledged.

\section{Appendix A. Expression of the piezoelectric coupling factor $\theta$}

In this appendix, the piezoelectric coupling factor $\theta$ of the lumped model (1) is derived from the virtual work of the stresses in 4 piezoelectric layers stacked symmetrically as in Figure 16(right). The virtual work $\delta W$ of the stresses is written as

$$
\delta W=(\theta v+k z) \delta z
$$

where $\delta z$ is a virtual variation of the displacement $z$ at the tip of the beam. As we consider a Euler Bernoulli beam model, the constitutive relation between the axial stress $\sigma_{1}$, the axial deformation $\epsilon_{1}$ and the electrostatic field component $-V_{, 3}$ is reduced to $[17]$

$$
\sigma_{1}=E_{p} \epsilon_{1}-e_{31} V_{, 3}
$$

The infinitesimal work $\delta W$ of the stresses is given by

$$
\delta W=\int_{\Omega} \sigma_{1} \delta \epsilon_{1} d x_{1} d x_{2} d x_{3}
$$

Since the Euler-Bernoulli displacement $u\left(x_{1}, x_{3}\right)$ is of the form

$$
u=\left(\begin{array}{c}
-z x_{3} \phi^{\prime}\left(x_{1}\right) \\
z \phi\left(x_{1}\right)
\end{array}\right),
$$

the strain $\epsilon_{1}$ is equal to

$$
\epsilon_{1}=-z x_{3} \phi^{\prime \prime}\left(x_{1}\right)
$$

so that

$$
\delta \epsilon_{1}=-x_{3} \phi^{\prime \prime}\left(x_{1}\right) \delta z
$$

Assuming that the electric field in the piezoelectric medium is uniform, we have

$$
-V_{, 3}=\frac{v}{4 h}
$$

Substituting (A.5-A.7) in (A.3) and comparing with (A.1), we obtain

$$
\theta=\frac{e_{31}}{2 h} \int_{\Omega_{p}} x_{3} \phi^{\prime \prime}\left(x_{1}\right) d x_{1} d x_{2} d x_{3}
$$

where $\Omega_{p}$ is the domain occupied by the piezoelectric material on top of the beam. Since the beam is clamped at $x_{1}=0$, the modal shape $\phi$ satisfies the relation $\phi_{1}(0)=0$. Therefore the expression (A.8) becomes

$$
\theta=\frac{W_{p} e_{31}}{2 h} \phi^{\prime}(l) \int x_{3} d x_{3}
$$

For the configuration represented in Figure 16(right), we obtain $\theta=e_{31} W_{p} \frac{e+3 h}{2} \phi^{\prime}(l)$. 


\section{References}

[1] E.K. Reilly, F. Burghardt, R. Fain, and P. Wright. Powering a wireless sensor node with a vibration-driven piezoelectric energy harvester. Smart Materials and Structures, 20:125006, 2011.

[2] C.B. Williams, A. Pavic, R.S. Crouch, and R.C. Woods. Feasibility study of vibration-electric generator for bridge vibration sensors. In Society for Experimental Mechanics, Inc, 16 th International Modal Analysis Conference., volume 2, pages 1111-1117, 1998.

[3] C.B. Williams and R.B. Yates. Analysis of a micro-electric generator for microsystems. Sensors and Actuators A: Physical, 52(1):8-11, 1996.

[4] N.G. Elvin, N. Lajnef, and A.A. Elvin. Feasibility of structural monitoring with vibration powered sensors. Smart materials and structures, 15:977, 2006.

[5] S.F. Ali, M.I. Friswell, and S. Adhikari. Analysis of energy harvesters for highway bridges. Journal of Intelligent Material Systems and Structures, 22(16):1929-1938, 2011.

[6] K.M. Farinholt, N. Miller, W. Sifuentes, J. MacDonald, G. Park, and C.R. Farrar. Energy harvesting and wireless energy transmission for embedded SHM sensor nodes. Structural Health Monitoring, 9(3):269-280, 2010.

[7] T.V. Galchev, J. McCullagh, R.L. Peterson, and K. Najafi. Harvesting traffic-induced vibrations for structural health monitoring of bridges. Journal of Micromechanics and Microengineering, 21:104005, 2011.

[8] D. Siegert, M. Estivin, J. Billo, F.X. Barin, and F. Toutlemonde. Extreme effect of traffic loads on a prestressed concrete bridge. In Proceeding of the International Conference on Heavy Vehicle, Paris, 2008.

[9] D. Siegert, L. Mevel, E. Reynders, G. De Roeck, and M. Goursat. Variation of modal parameter estimates of a prestressed concrete bridge. In 27th International Modal Analysis Conference (IMAC-XXVII), 2009.

[10] E. Balmes, M. Corus, D. Siegert, et al. Modeling thermal effects on bridge dynamic responses. In Proceedings of the 24th International Modal Analysis Conference (IMAC-XXIV), 2006.

[11] S. Priya and D.J. Inman. Energy harvesting technologies. Springer Verlag, 2009.

[12] CAST3M homepage. www-cast3m.cea.fr.

[13] N.E. Dutoit, L.W. Brian, and K. Sang-Gook. Design considerations for MEMS-scale piezoelectric mechanical vibration energy harvesters. Integrated Ferroelectrics, 71(1):121-160, 2005.

[14] A.W. Drake. Fundamentals of Applied Probability Theory. McGraw-Hill, New York, 1988.

[15] D.F. Mix. Random Signal Processing. Prentice Hall, Englewood Cliffs, 1995.

[16] J. Carson. The statistical energy-frequency system spectrum of random disturbances. Bell Syst. Tech. J, 10:374-381, 1931.

[17] IEEE Standard on Piezoelectricity. New York IEEE, 1987. 